\title{
NUMERICAL METHOD FOR COUPLING THE MACRO AND MESO SCALES IN STOCHASTIC CHEMICAL KINETICS
}

\author{
LARS FERM ${ }^{1}$, PER LÖTSTEDT ${ }^{1}$ \\ ${ }^{1}$ Division of Scientific Computing, Department of Information Technology \\ Uppsala University, SE-75105 Uppsala, Sweden \\ emails: perl, ferm Qit.uu.se
}

\begin{abstract}
A numerical method is developed for simulation of stochastic chemical reactions. The system is modeled by the Fokker-Planck equation for the probability density of the molecular state. The dimension of the domain of the equation is reduced by assuming that most of the molecular species have a normal distribution with a small variance. The numerical approximation preserves properties of the analytical solution such as nonnegativity and constant total probability. The method is applied to a nine dimensional problem modelling an oscillating molecular clock. The oscillations stop at a fixed point with a macroscopic model but they continue with our two dimensional, mixed macroscopic and mesoscopic model.
\end{abstract}

Keywords: Fokker-Planck equation, reaction rate equations, dimensional reduction, stochastic chemical kinetics

AMS subject classification: $65 \mathrm{M} 20,65 \mathrm{M} 60$

\section{Introduction}

Deterministic models for chemical reactions are inaccurate in describing the dynamics of the chemical systems when the copy numbers of the reacting molecules are small or the system is in the neighborhood of an instability [16]. When only a few molecules participate in the reactions, the molecular fluctuations dominate the behavior of the system and the deterministic and macroscopic reaction rate equations for the concentrations of the chemical species are not sufficiently precise. The need for a mesoscopic, probabilistic view is especially important in certain models for the biochemistry of living cells [6, 20, 21, 27]. A stochastic 
model equation is the chemical master equation [16] for the probability of a well stirred chemical system to be in a particular state.

The master equation is a scalar differential-difference equation for the probability of the system to be in a certain state at time $t$. The computational difficulty with this equation is that the number of dimensions of the problem and the state vector grows with the number of molecular species in the model. The standard method for solution of the high dimensional problems is the Stochastic Simulation Algorithm (SSA) by Gillespie [10,11]. Each reaction is simulated and the integer states of the involved species are updated accordingly. Two random numbers determine when the next reaction takes place and which reaction it is. The advantage of SSA is that the computational work increases slowly with the dimension of the problem compared to the exponential growth with a deterministic method for the master equation. A disadvantage of the method is that the time steps between the reactions can be very small compared to the prevailing dynamics of the system if there are separate time scales in the solution. The fast scale requires small time increments but for an accurate solution it is sufficient to follow the slow scale. Different ways of circumventing this problem based on an approximate treatment of the fast scale are found in $[4,15,21]$.

The Fokker-Planck equation (FPE) is a partial differential equation (PDE) approximating the master equation $[2,16,23]$. The state of the system is assumed to change continuously and common numerical methods for PDEs can be invoked for solution of the FPE. The computational complexity is mitigated somewhat but the problem with exponential growth in work and memory remains. In [18] the dimension of the FPE is reduced by assuming that the major part of the molecular species are normally distributed with a small variance and that only a small set of species needs a full stochastic treatment with an FPE. The dimension reduction in [18] is applicable also to the master equation. Equations for the expected values of the majority of the species are derived and they are coupled to one FPE for the probability density function (PDF) in a low dimension. The equations for the expected values are PDEs similar to the FPE or integro-differential equations (IDEs) resembling the macroscopic reaction rate equations. A numerical scheme for the coupled equations is derived and tested in this paper.

The PDEs of FPE type are discretized in space by a finite volume method. The PDEs preserve the total probability and the non-negativity of the solutions and the numerical method shares these properties. The expected values are also non-negative in the numerical solution if they are so in the analytical solution. If the solutions are allowed to be negative, then severe numerical instabilities occur. The solution is advanced in time by an unconditionally stable implicit method and thus problems with stiff systems are avoided. If the space discretization is stable, then there is a unique and positive steady state solution. The method is applied to a model in $[1,28]$ for the circadian rhythm including nine species. The 9D scalar problem is reduced to the solution of two PDEs in two dimensions and six nonlinear IDEs. Most of the species satisfy equations similar to the reaction 
rate equations and only a few are modeled stochastically. This is an example where the macroscale model fails to reproduce the dynamics of the system. The result is sensitive to a parameter in the model. When it has a high value, then our system of equations and the reaction rate equations both exhibit an oscillatory behavior. With a low value, the macroscopic model terminates at a fixed point while the partly stochastic model continues to oscillate.

The outline of the paper is as follows. In the next section, the reduced system of equations from [18] is given. The equations are discretized in space and time and the boundary conditions are stated in Section 3. The properties of the discrete solution are compared to the properties of the analytical solution in Section 4. The equations for the reduced stochastic model for the molecular clock in $[1,28]$ are solved in Section 5 and compared to the solution of the reaction rate equations. Finally, conclusions are drawn in the last section.

The $i$ :th element of a vector $\mathbf{v}$ is denoted by $v_{i}$. If $v_{i} \geq 0$ for all $i$, then we write $\mathbf{v} \geq 0$. The $\ell_{p}$-norm of $\mathbf{v}$ of length $N$ then is $\|\mathbf{v}\|_{p}=\left(\sum_{i=1}^{N}\left|v_{i}\right|^{p}\right)^{1 / p}$ in the paper.

\section{The system of equations}

With $N$ chemically active molecular species $X_{i}, i=1, \ldots, N, x_{i}$ denotes the number of molecules of substrate $X_{i}$. The state vector of the chemical system is $\mathbf{x}$. A reaction $r$ is a transition from a state $\mathbf{x}_{r}$ to $\mathbf{x}$ so that $\mathbf{x}_{r}=\mathbf{x}+\mathbf{n}_{r}$. There is a non-negative propensity $w_{r}\left(\mathbf{x}_{r}, t\right)$ for the probability of the reaction to occur per unit time. The reaction $r$ can now be written

$$
\mathbf{x}_{r} \stackrel{w_{r}\left(\mathbf{x}_{r}, t\right)}{\longrightarrow} \mathbf{x}, \mathbf{n}_{r}=\mathbf{x}_{r}-\mathbf{x} .
$$

The time evolution of the state of the chemical system is governed by the master equation [16]. The master equation is a differential-difference equation for the PDF $p(\mathbf{x}, t)$ for the system to be in the state $\mathbf{x}$ at time $t$. The PDF is non-negative for all $\mathbf{x}$ and $t$. The FPE is a PDE for $p$ approximating the master equation $[16,23]$ for $\mathbf{x} \in \mathbb{R}_{+}^{N}$, the non-negative $N$-dimensional orthant, and $t \geq 0$.

In [18], the state vector is split into two parts $\mathbf{x}^{T} \rightarrow\left(\mathbf{x}^{T}, \mathbf{y}^{T}\right)$ with $\mathbf{x} \in \mathbb{R}_{+}^{m}$ and $\mathbf{y} \in \mathbb{R}^{n}$. The transition vector $\mathbf{n}_{r}$ for reaction $r$ is split in the same manner $\mathbf{n}_{r}^{T} \rightarrow$ $\left(\mathbf{m}_{r}^{T}, \mathbf{n}_{r}^{T}\right)$ with $\mathbf{m}_{r} \in \mathbb{Z}_{+}^{m}, \mathbf{n}_{r} \in \mathbb{Z}_{+}^{n}$, where $\mathbb{Z}_{+}$denotes the non-negative integer numbers. The corresponding sets of stochastic variables are $X_{i}, i=1, \ldots, m$, and $Y_{i}, i=1, \ldots, n$. The assumption is that given $\mathbf{X}=\mathbf{x}$ the stochastic variables $Y_{i}$ are mutually independent and normally distributed with a small variance. The $\mathrm{PDF}$ of the full system is

$$
p(\mathbf{x}, \mathbf{y}, t)=\left(2 \sigma^{2} \pi\right)^{-n / 2} p_{0}(\mathbf{x}, t) \exp \left(-\sum_{j=1}^{n} \frac{\left(y_{j}-\phi_{j}\right)^{2}}{2 \sigma^{2}}\right), \mathbf{x} \in \mathbb{R}_{+}^{m}, \mathbf{y} \in \mathbb{R}^{n} .
$$


Assuming that the variance $\sigma$ in (2) is small, a PDE is derived in [18] for the first partial moment for $y_{k}$ with respect to $\mathbf{y}$. This moment is

$$
p_{k}(\mathbf{x}, t)=\int_{\mathbb{R}^{n}} y_{k} p(\mathbf{x}, \mathbf{y}, t) d \mathbf{y}=p_{0}(\mathbf{x}, t) \phi_{k}(\mathbf{x}, t) .
$$

The conditional expected value of $y_{k}$ is $\phi_{k}$ since

$$
E\left[y_{k} \mid \mathbf{x}\right]=p_{k}(\mathbf{x}, t) / p_{0}(\mathbf{x}, t)=\phi_{k}(\mathbf{x}, t) .
$$

The PDE for $p_{k}$ is a FPE with a source term

$$
\frac{\partial p_{k}(\mathbf{x}, t)}{\partial t}=\sum_{r=1}^{R} \nabla \cdot \mathbf{F}_{r k}-\sum_{r=1}^{R} n_{r k} w_{r}(\mathbf{x}, \boldsymbol{\phi}(\mathbf{x}, t), t) p_{0}(\mathbf{x}, t),
$$

for a chemical system with $R$ reactions, where $\mathbf{F}_{r k}$ is defined by

$$
F_{r k i}=m_{r i}\left(q_{r k}+0.5 \mathbf{m}_{r} \cdot \nabla q_{r k}\right), i=1, \ldots, m, k=1, \ldots, \nu, r=1, \ldots, R,
$$

and $q_{r k}(\mathbf{x}, t)=w_{r}(\mathbf{x}, \phi, t) p_{k}(\mathbf{x}, t)$.

If the difference between $\phi_{k}$ and its expected value

$$
\bar{\phi}_{k}=\int_{\mathbb{R}_{+}^{m}} \phi_{k}(\mathbf{x}, t) p_{0}(\mathbf{x}, t) d \mathbf{x}=\int_{\mathbb{R}_{+}^{m}} p_{k}(\mathbf{x}, t) d \mathbf{x}=\int_{\mathbb{R}^{n}} \int_{\mathbb{R}_{+}^{m}} y_{k} p(\mathbf{x}, \mathbf{y}, t) d \mathbf{x} d \mathbf{y}
$$

is small for $k>\nu$, then an IDE is derived in [18] for $\bar{\phi}_{k}, k=\nu+1, \ldots, n$,

$$
\frac{d \bar{\phi}_{k}}{d t}=-\sum_{r=1}^{R} n_{r k} \int_{\mathbb{R}_{+}^{m}} w_{r}(\mathbf{x}, \boldsymbol{\phi}(\mathbf{x}, t), t) p(\mathbf{x}, t) d \mathbf{x} .
$$

Now $\phi$ is defined by

$$
\boldsymbol{\phi}^{T}=\left(\phi_{1}(\mathbf{x}, t), \ldots, \phi_{\nu}(\mathbf{x}, t), \bar{\phi}_{\nu+1}(t), \ldots, \bar{\phi}_{n}(t)\right) .
$$

The shift in $\mathbf{x}$ by $\mathbf{m}_{r}$ is ignored in (5) and (7) compared to the version in [18].

The reaction rate equations corresponding to (7) are a system of nonlinear ordinary differential equations (ODEs)

$$
\frac{d \bar{\phi}_{k}}{d t}=-\sum_{r=1}^{R} n_{r k} w_{r}(\bar{\phi}(t), t)
$$

The FPE for $p_{0}$ is

$$
\frac{\partial p_{0}(\mathbf{x}, t)}{\partial t}=\sum_{r=1}^{R}\left\{\sum_{i=1}^{m} m_{r i} \frac{\partial q_{r}(\mathbf{x}, t)}{\partial x_{i}}+0.5 \sum_{i=1}^{m} \sum_{j=1}^{m} m_{r i} m_{r j} \frac{\partial^{2} q_{r}(\mathbf{x}, t)}{\partial x_{i} \partial x_{j}}\right\}
$$


with $q_{r}(\mathbf{x}, t)=w_{r}(\mathbf{x}, \boldsymbol{\phi}, t) p_{0}(\mathbf{x}, t)$. This is a PDE to be solved for the scalar $p_{0}$ in $m$ dimensions. The number of dimensions $m$ should be kept small so that the problem is computationally tractable. Alternatively, the equation for $p_{0}$ can be written

$$
\begin{aligned}
\frac{\partial p_{0}(\mathbf{x}, t)}{\partial t}=\sum_{r=1}^{R}\left(\mathbf{m}_{r} \cdot \nabla\right)\left(q_{r}+0.5\left(\mathbf{m}_{r} \cdot \nabla\right) q_{r}\right) \\
=\sum_{r=1}^{R} \begin{array}{l}
0.5 w_{r}\left(\mathbf{m}_{r} \cdot \nabla\right)^{2} p_{0}+\left(w_{r}+\left(\mathbf{m}_{r} \cdot \nabla\right) w_{r}\right)\left(\mathbf{m}_{r} \cdot \nabla\right) p_{0} \\
\\
+p_{0}\left(\mathbf{m}_{r} \cdot \nabla\right)\left(w_{r}+0.5\left(\mathbf{m}_{r} \cdot \nabla\right) w_{r}\right)
\end{array}
\end{aligned}
$$

The same FPE in conservation form is

$$
\frac{\partial p_{0}(\mathbf{x}, t)}{\partial t}=\sum_{r=1}^{R} \nabla \cdot \mathbf{F}_{r}=\nabla \cdot \mathbf{F}
$$

with the flux functions

$$
F_{r i}=m_{r i}\left(q_{r}+0.5 \mathbf{m}_{r} \cdot \nabla q_{r}\right), i=1, \ldots, m, r=1, \ldots, R .
$$

The boundary conditions at $x_{i}=0, i=1, \ldots, N$, are

$$
F_{i}=\sum_{r=1}^{R} F_{r i}=\sum_{r=1}^{R} m_{r i}\left(p_{0}\left(w_{r}+0.5\left(\mathbf{m}_{r} \cdot \nabla\right) w_{r}\right)+0.5 w_{r}\left(\mathbf{m}_{r} \cdot \nabla\right) p_{0}\right)=0 .
$$

The equations $(5)$ and $(9,10,11)$ are solved numerically in $\bar{\Omega}_{h}$, where $\Omega_{h}=$ $\left\{\mathbf{x} \mid 0<x_{i}<x_{\max }\right\}$ is an open domain and $x_{\max }$ is so large that $p \approx 0$ and $\nabla p \approx 0$ in the neighborhood of $x_{i}=x_{\max }$ for all $i$. If $p=0$ and $\nabla p=0$ at $x_{i}=x_{\max }$ for all $i$, then condition (12) is also satisfied there. The FPE (11) and the boundary conditions imply that the total probability for $p_{0}$

$$
P_{0}(t)=\int_{\Omega_{h}} p_{0}(\mathbf{x}, t) d \mathbf{x}
$$

is constant for $t \geq 0$, see [8].

\section{Discretization of the Fokker-Planck equation}

In order to conserve the total probability, the FPEs in conservation form in (5) and (11) are discretized by a finite volume method in space. The grid is Cartesian covering $\bar{\Omega}_{h}$ with cells $\omega$. The trapezoidal method (or Crank-Nicolson method) advances the solution in time from $t^{l}$ to $t^{l+1}$ with the time step $\Delta t$. The coefficients in the difference stencil are such that the non-negativity of the PDF is preserved. 


\subsection{Space discretization}

The space operator in (5) and (9) is written with $p=p_{0}$ or $p=p_{k}$

$$
\begin{aligned}
\nabla \cdot \sum_{r=1}^{R} \mathbf{F}_{r} & =\sum_{i=1}^{m} \frac{\partial}{\partial x_{i}}\left(\sum_{r=1}^{R} m_{r i} w_{r}\right) p+0.5 \sum_{i=1}^{m} \frac{\partial}{\partial x_{i}}\left(\sum_{r=1}^{R} \sum_{j=1}^{m} m_{r i} m_{r j} \frac{\partial w_{r} p}{\partial x_{j}}\right) \\
& =\nabla \cdot \mathbf{G}+0.5 \nabla \cdot \mathbf{H},
\end{aligned}
$$

where

$$
G_{i}=\left(\sum_{r=1}^{R} m_{r i} w_{r}\right) p, H_{i}=\sum_{r=1}^{R} H_{r i}=\sum_{r=1}^{R} \sum_{j=1}^{m} m_{r i} m_{r j} \frac{\partial w_{r} p}{\partial x_{j}} .
$$

The FPE is integrated over a cell $\omega$ and the time interval $\left[t^{l}, t^{l+1}\right]$

$$
\int_{t^{l}}^{t^{l+1}} \int_{\omega} \frac{\partial p}{\partial t} d \omega d t=\int_{t^{l}}^{t^{l+1}} \int_{\omega} \nabla \cdot \mathbf{G}+0.5 \nabla \cdot \mathbf{H} d \omega d t .
$$

The average $\bar{p}$ of $p$ in $\omega$ satisfies

$$
\bar{p}^{l+1}-\bar{p}^{l}=|\omega|^{-1} \int_{t^{l}}^{t^{l+1}} \int_{\omega} \nabla \cdot \mathbf{G}+0.5 \nabla \cdot \mathbf{H} d \omega d t,
$$

where $|\omega|$ is the area of $\omega$.

The formulas are simplified if the approximations are derived for two space dimensions, $m=2$, and a constant step size, but the generalizations to higher dimensions and variable step sizes are straightforward. The integral form of (14) is taken over a square cell $\omega_{i j}$ with edge length $h$ with midpoint at $\left(x_{1 i j}, x_{2 i j}\right)$ and corners at $\left(x_{1 i j} \pm h / 2, x_{2 i j} \pm h / 2\right)$. The four faces of $\omega_{i j}$ are denoted in the counterclockwise direction by $\partial \omega_{1 i j}\left(\right.$ where $\left.x_{1}=x_{1 i j}+h / 2\right), \partial \omega_{2 i j}\left(x_{2}=x_{2 i j}+h / 2\right)$, $\partial \omega_{3 i j}\left(x_{1}=x_{1 i j}-h / 2\right)$, and $\partial \omega_{4 i j}\left(x_{2}=x_{2 i j}-h / 2\right)$. Then

$$
\begin{aligned}
& \int_{\omega_{i j}} \nabla \cdot \mathbf{G}+0.5 \nabla \cdot \mathbf{H} d \omega= \\
& \int_{\partial \omega_{1 i j}} G_{1}+0.5 H_{1} d x_{2}-\int_{\partial \omega_{3 i j}} G_{1}+0.5 H_{1} d x_{2} \\
& +\int_{\partial \omega_{2 i j}} G_{2}+0.5 H_{2} d x_{1}-\int_{\partial \omega_{4 i j}} G_{2}+0.5 H_{2} d x_{1}
\end{aligned}
$$

by Gauss' integral formula. We need evaluations of $G_{i}+0.5 H_{i}$ on the four edges of the cell in a finite volume discretization.

Let $\bar{w}_{i}=\sum_{r} m_{r i} w_{r}, i=1,2$, and let $p_{i j}$ be the approximation of $\bar{p}$ in cell $\omega_{i j}$. Then $\mathbf{G}$ is approximated as follows at $x_{1}=x_{1 i j}+h / 2$

$$
G_{1}=\bar{w}_{1} p \approx \begin{cases}\bar{w}_{1, i+1 / 2, j} p_{i+1, j}, & \bar{w}_{1, i+1 / 2, j} \geq 0, \\ \bar{w}_{1, i+1 / 2, j} p_{i j}, & \bar{w}_{1, i+1 / 2, j}<0,\end{cases}
$$


and at $x_{2}=x_{2 i j}+h / 2$

$$
G_{2}=\bar{w}_{2} p \approx \begin{cases}\bar{w}_{2, i, j+1 / 2} p_{i, j+1}, & \bar{w}_{2, i, j+1 / 2} \geq 0 \\ \bar{w}_{2, i, j+1 / 2} p_{i j}, & \bar{w}_{2, i, j+1 / 2}<0\end{cases}
$$

where

$$
\begin{aligned}
& \bar{w}_{1, i+1 / 2, j}=\bar{w}_{1}\left(\mathbf{x}_{i+1 / 2, j}, \boldsymbol{\phi}\left(\mathbf{x}_{i+1 / 2, j}, t\right), t\right), \mathbf{x}_{i+1 / 2, j}=\mathbf{x}_{i j}+(h / 2,0)^{T}, \\
& \bar{w}_{2, i, j+1 / 2}=\bar{w}_{2}\left(\mathbf{x}_{i, j+1 / 2}, \boldsymbol{\phi}\left(\mathbf{x}_{i, j+1 / 2}, t\right), t\right), \mathbf{x}_{i, j+1 / 2}=\mathbf{x}_{i j}+(0, h / 2)^{T} .
\end{aligned}
$$

The flux $\mathbf{G}$ at $x_{1}=x_{1 i j}-h / 2$ and $x_{2}=x_{2 i j}-h / 2$ is determined in the same manner. After division by the area $|\omega|=h^{2}$ of the cell, the first derivatives in the first sum in (14) are discretized by a first order accurate upwind approximation depending on the sign of $\bar{w}_{i}$ on the face between two cells.

The second derivatives are computed as follows for $\left|m_{r i}\right| \leq 1$. In two dimensions, the $\mathbf{H}_{r}$-fluxes are

$$
H_{r 1}=m_{r 1}^{2} \frac{\partial q_{r}}{\partial x_{1}}+m_{r 1} m_{r 2} \frac{\partial q_{r}}{\partial x_{2}}, H_{r 2}=m_{r 1} m_{r 2} \frac{\partial q_{r}}{\partial x_{1}}+m_{r 2}^{2} \frac{\partial q_{r}}{\partial x_{2}} .
$$

Let $s_{r}=m_{r 1} m_{r 2}$ and let $c_{i j}$ be values in the corners of the cell defined by

$$
c_{i+1 / 2, j+1 / 2}= \begin{cases}q_{r, i+1, j+1}-q_{r i j}, & s_{r}=1, \\ q_{r, i+1, j}-q_{r, i, j+1}, & s_{r}=-1,\end{cases}
$$

where $q_{r i j}=w_{r}\left(\mathbf{x}_{i j}, \boldsymbol{\phi}\left(\mathbf{x}_{i j}, t\right), t\right) p_{i j}=w_{r i j} p_{i j}$. Then $H_{r k}, k=1,2$, at $\partial \omega_{1 i j}$ and $\partial \omega_{2 i j}$ is approximated by

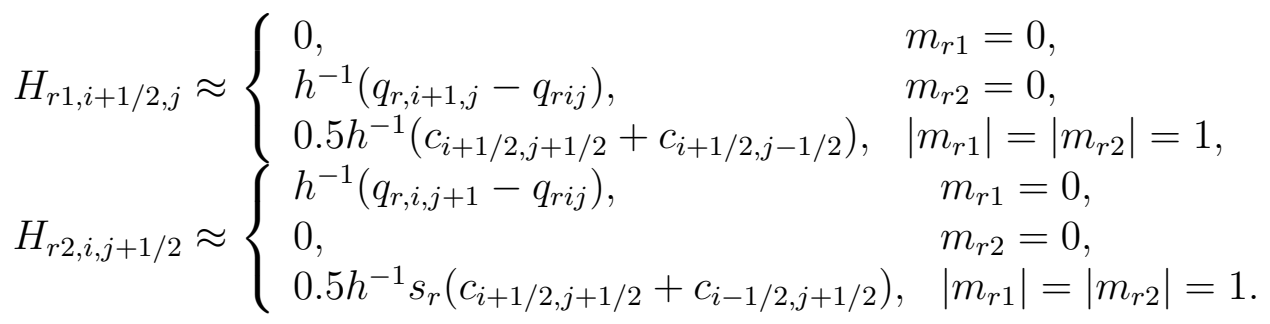

For cell $\omega_{i j}$ and $\left|s_{r}\right|=1$, the sum of the four integrated $\mathbf{H}$-fluxes for reaction $r$ is

$$
\begin{aligned}
0.5 h^{-1}(\quad & c_{i+1 / 2, j+1 / 2}+c_{i+1 / 2, j-1 / 2}-c_{i-1 / 2, j+1 / 2}-c_{i-1 / 2, j-1 / 2} \\
& \left.+s_{r} c_{i+1 / 2, j+1 / 2}-s_{r} c_{i+1 / 2, j-1 / 2}+s_{r} c_{i-1 / 2, j+1 / 2}-s_{r} c_{i-1 / 2, j-1 / 2}\right) \\
=0.5 h^{-1}( & \left(1+s_{r}\right) c_{i+1 / 2, j+1 / 2}-\left(1+s_{r}\right) c_{i-1 / 2, j-1 / 2} \\
& \left.+\left(1-s_{r}\right) c_{i+1 / 2, j-1 / 2}-\left(1-s_{r}\right) c_{i-1 / 2, j+1 / 2}\right) .
\end{aligned}
$$

After division by the area of $\omega_{i j}$, the resulting formula is

$$
\left|\omega_{i j}\right|^{-1} \int_{\omega_{i j}} \nabla \cdot \mathbf{H}_{r} d \omega \approx \begin{cases}h^{-2}\left(q_{r, i+1, j+1}-2 q_{r i j}+q_{r, i-1, j-1}\right), & s_{r}=1 \\ h^{-2}\left(q_{r, i+1, j-1}-2 q_{r i j}+q_{r, i-1, j+1}\right), & s_{r}=-1 .\end{cases}
$$


Regarded as a finite difference method for the PDF $p_{i j}$ at the midpoints in a cell, the approximations (19), (20), of the first derivatives in (5) and (9) on a grid with constant grid size $h$ is first order accurate and in two dimensions

$$
\frac{\partial \bar{w}_{k} p}{\partial x_{i}} \approx \begin{cases}h^{-1}\left(\bar{w}_{k, i+1 / 2, j} p_{i+1, j}-\bar{w}_{k, i-1 / 2, j} p_{i j}\right), & \bar{w}_{k, i+1 / 2, j}, \bar{w}_{k, i-1 / 2, j} \geq 0, \\ h^{-1}\left(\bar{w}_{k, i+1 / 2, j} p_{i j}-\bar{w}_{k, i-1 / 2, j} p_{i-1, j}\right), & \bar{w}_{k, i+1 / 2, j}, \bar{w}_{k, i-1 / 2, j}<0, \\ h^{-1}\left(\bar{w}_{k, i+1 / 2, j} p_{i+1, j}-\bar{w}_{k, i-1 / 2, j} p_{i-1, j}\right), & \bar{w}_{k, i+1 / 2, j} \geq 0, \bar{w}_{k, i-1 / 2, j}<0, \\ h^{-1}\left(\bar{w}_{k, i+1 / 2, j}-\bar{w}_{k, i-1 / 2, j}\right) p_{i j}, & \bar{w}_{k, i+1 / 2, j}<0, \bar{w}_{k, i-1 / 2, j} \geq 0 .\end{cases}
$$

The coefficients multiplying $p_{k l}$ are non-negative except for the cell in the center which has a non-positive coefficient. The second derivatives multiplied by the coefficents $m_{r i} m_{r j}$ in (9) are approximated as in (22), (23), by

$$
\begin{aligned}
& \sum_{i, j=1}^{2} m_{r i} m_{r j} \frac{\partial^{2} w_{r} p}{\partial x_{i} \partial x_{j}} \approx \\
& h^{-2}\left(w_{r, i+m_{r i}, j+m_{r j}} p_{i+m_{r i}, j+m_{r j}}-2 w_{r i j} p_{i j}+w_{r, i-m_{r i}, j-m_{r j}} p_{i-m_{r i}, j-m_{r j}}\right) .
\end{aligned}
$$

This is a second order accurate approximation of the sum also when $\left|m_{r i}\right|$ or $\left|m_{r j}\right|$ is greater than one. If $w_{r} \geq 0$, then all coefficients in the stencil multiplying $p_{k l}$ are non-negative except for $p_{i j}$ in $\omega_{i j}$.

The integral of the source term in (5) is the expected value of $w_{r}$ in $\omega_{i j}$. It is approximated by the rectangle rule

$$
\int_{\omega_{i j}} w_{r}(\mathbf{x}, \boldsymbol{\phi}(\mathbf{x}, t), t) p_{0}(\mathbf{x}, t) d \omega \approx\left|\omega_{i j}\right| w_{r}\left(\mathbf{x}_{i j}, \boldsymbol{\phi}\left(\mathbf{x}_{i j}, t\right), t\right) p_{0 i j} .
$$

The integral in (7) is obtained by summing over all cells in (26).

\subsection{Boundary conditions and conservation}

Consider one rectangular cell $\omega_{i j}$ in the grid of length $h_{i}$ in the $x$-direction and $h_{j}$ in the $y$-direction. The integral of the space operator over $\omega_{i j}$ is written according to the approximations above

$$
\begin{aligned}
& \left|\omega_{i j}\right|^{-1} \int_{\omega_{i j}} \nabla \cdot \mathbf{G}+0.5 \nabla \cdot \mathbf{H} d \omega= \\
& h_{i}^{-1}\left(F_{1, i+1 / 2, j}-F_{1, i-1 / 2, j}\right)+h_{j}^{-1}\left(F_{2, i, j+1 / 2}-F_{2, i, j-1 / 2}\right),
\end{aligned}
$$

with $F_{k, i, j}=\left(G_{k}+0.5 H_{k}\right)_{i j}$ and $i, j=1, \ldots, L$. In the boundary cells of the computational domain, the total flux $\mathbf{G}+0.5 \mathbf{H}$ is zero at a cell face on the boundary according to the boundary conditions of the differential equation (12). At the 
left and right boundaries of the domain, e.g. at $x_{1}=0$ we have $F_{1,1 / 2, j}=0$, and at $x_{1}=x_{\max }, F_{1, L+1 / 2, j}=0$. The area weighted sum of the spatial approximation over all cells vanishes

$$
\begin{aligned}
& \sum_{i=1}^{L} \sum_{j=1}^{L}\left|\omega_{i j}\right| h_{i}^{-1}\left(F_{1, i+1 / 2, j}-F_{1, i-1 / 2, j}\right)+h_{j}^{-1}\left(F_{2, i, j+1 / 2}-F_{2, i, j-1 / 2}\right)= \\
& \sum_{j=1}^{L} h_{j}\left(F_{1, L+1 / 2, j}-F_{1,1 / 2, j}\right)+\sum_{i=1}^{L} h_{i}\left(F_{2, i, L+1 / 2}-F_{2, i, 1 / 2}\right)=0
\end{aligned}
$$

thanks to the conservative formulation and the boundary conditions. This property is shared by the analytical solution in (11) since

$$
\int_{\bar{\Omega}_{h}} \nabla \cdot \mathbf{F} d \Omega=\int_{\partial \Omega_{h}} \mathbf{F} \cdot \mathbf{n}_{\Omega} d S=0
$$

where $\partial \Omega_{h}$ is the boundary of $\bar{\Omega}_{h}$ and $\mathbf{n}_{\Omega}$ is the normal of $\partial \Omega_{h}$ (cf. (13)).

The approximation in a cell at the left boundary in a two-dimensional domain requires one extra numerical condition in a column of ghost cells with index $i=0$. The space operator in $\omega_{1 j}$ is

$$
\begin{aligned}
& F_{1,3 / 2, j}+F_{2,1, j+1 / 2}-F_{2,1, j-1 / 2} \\
& =G_{1,3 / 2, j}+G_{2,1, j+1 / 2}-G_{2,1, j-1 / 2}+0.5\left(H_{1,3 / 2, j}+H_{2,1, j+1 / 2}-H_{2,1, j-1 / 2}\right) .
\end{aligned}
$$

The evaluations of $G_{r k}$ and $H_{r k}$ when $s_{r}=0$ for reaction $r$ pose no problem at the boundary but for $s_{r} \neq 0$ we have

$$
H_{r 1,3 / 2, j}+H_{r 2,1, j+1 / 2}-H_{r 2,1, j-1 / 2}=\left\{\begin{array}{l}
c_{3 / 2, j+1 / 2}-0.5 c_{1 / 2, j-1 / 2}+0.5 c_{1 / 2, j+1 / 2} \\
\text { when } s_{r}=1, \\
c_{3 / 2, j-1 / 2}-0.5 c_{1 / 2, j+1 / 2}+0.5 c_{1 / 2, j-1 / 2} \\
\text { when } s_{r}=-1 .
\end{array}\right.
$$

By letting $q_{r 0 j}=q_{r 1 j}$ in the ghost cell, the stencil in (23)

$$
\left|\omega_{1 j}\right|^{-1} \int_{\omega_{1 j}} \nabla \cdot \mathbf{H}_{r} d \omega \approx\left\{\begin{array}{l}
h^{-2}\left(q_{r, 2, j+1}-2 q_{r 1 j}+0.5\left(q_{r, 1, j+1}+q_{r, 1, j-1}\right)\right) \\
\text { when } s_{r}=1 \\
h^{-2}\left(q_{r, 2, j-1}-2 q_{r 1 j}+0.5\left(q_{r, 1, j+1}+q_{r, 1, j-1}\right)\right) \\
\text { when } s_{r}=-1
\end{array}\right.
$$

The other boundaries are treated in the same manner. If $w_{r} \geq 0$, then all coefficients in the stencil multiplying $p_{k l}$ are non-negative also at the boundaries except for $p_{1 j}$ in $\omega_{1 j}$.

A generalization of the approximations (22) to the case when $\left|m_{r i}\right|,\left|m_{r j}\right|>1$, is possible, but the treatment of the boundaries and conservation of the total probability is more complicated. The resulting stencil in the interior of the domain is still given by (25). 


\subsection{Time discretization}

The mean values of $p_{0}$ and $p_{k}, k=1, \ldots, \nu$, in the cells and the mean values $\bar{\phi}_{k}, k=\nu+1, \ldots, n$, in $\bar{\Omega}_{h}$ are advanced in time by the trapezoidal method [14] applied to (11), (5), and (7).

Let $\mu$ be a multi-index such that $\mu=\left(\mu_{1}, \mu_{2}, \ldots, \mu_{m}\right)$. Then $p_{i \mu}^{l}, i=0,1, \ldots, \nu$, approximates the mean value of $p_{i}\left(\mathbf{x}, t^{l}\right)$ in cell $\omega_{\mu}$. The solution vector $\mathbf{p}_{i}^{l}$ consists of $p_{i \mu}^{l}$ in some order. After time discretization, (11) is

$$
\mathbf{p}_{0}^{l+1}-\mathbf{p}_{0}^{l}=0.5 \Delta t\left(A^{l+1} \mathbf{p}_{0}^{l+1}+A^{l} \mathbf{p}_{0}^{l}\right),
$$

where $A^{l}$ is a sparse matrix representing the space discretization in Section 3.1 and depends on $\mathbf{x}_{\mu}, \phi^{l}$, and $t^{l}$. Since $\phi_{k}(\mathbf{x}, t)=p_{k}(\mathbf{x}, t) / p_{0}(\mathbf{x}, t)$, the elements of $A^{l}$ depend nonlinearly on $\mathbf{p}_{0}^{l}$ and $\mathbf{p}_{k}^{l}$. In cell $\mu, \phi_{k \mu}^{l}$ is computed by

$$
\phi_{k \mu}^{l}=\phi_{k}\left(\mathbf{x}_{\mu}, t^{l}\right)=p_{k \mu}^{l} / p_{0 \mu}^{l} \text {. }
$$

Introduce the diagonal matrix $B_{k}^{l}$ defined by

$$
\left.\left(B_{k}^{l} \mathbf{p}_{0}^{l}\right)_{\mu}=\sum_{r=1}^{R} n_{r k} w_{r}\left(\mathbf{x}_{\mu}, \boldsymbol{\phi}_{\mu}^{l}, t\right), t\right) p_{0 \mu}^{l} .
$$

Then (5) for $k=1, \ldots, \nu$, is approximated in time by

$$
\mathbf{p}_{k}^{l+1}-\mathbf{p}_{k}^{l}=0.5 \Delta t\left(A^{l+1} \mathbf{p}_{k}^{l+1}+A^{l} \mathbf{p}_{k}^{l}\right)-0.5 \Delta t\left(B_{k}^{l+1} \mathbf{p}_{0}^{l+1}+B_{k}^{l} \mathbf{p}_{0}^{l}\right) .
$$

The matrix $A^{l}$ multiplying $\mathbf{p}_{0}^{l}$ in (29) and $\mathbf{p}_{k}^{l}$ in (32) is the same. Also $B_{k}^{l}$ depends in a nonlinear way on $\mathbf{p}_{0}^{l}$ and $\mathbf{p}_{k}^{l}$.

The areas of the cells $\left|\omega_{\mu}\right|$ are collected in the vector $\boldsymbol{\omega}$ in the same order as in $\mathbf{p}_{0}$. Then the discretization of the IDEs (7) is

$$
\bar{\phi}_{k}^{l+1}-\bar{\phi}_{k}^{l}=-0.5 \Delta t\left(\boldsymbol{\omega}^{T} B_{k}^{l+1} \mathbf{p}_{0}^{l+1}+\boldsymbol{\omega}^{T} B_{k}^{l} \mathbf{p}_{0}^{l}\right)
$$

for $k=\nu+1, \ldots, n$, using the rectangle rule (26). By conservation (27) (see also [8]), $\boldsymbol{\omega}^{T} A^{l}=0$ for all $l$. With $\bar{\phi}_{k}^{l}=\boldsymbol{\omega}^{T} \mathbf{p}_{k}^{l}$ we find that (33) is obtained by multiplying (32) from the left by $\boldsymbol{\omega}^{T}$. These operations correspond on the analytical side to the integration in (6) and the integration of (5) to arrive at (7).

The total discrete probability of $p_{0}$ is (cf. (13))

$$
P_{0}^{l+1}=\boldsymbol{\omega}^{T} \mathbf{p}_{0}^{l+1}=\boldsymbol{\omega}^{T}\left(\mathbf{p}_{0}^{l}+0.5 \Delta t\left(A^{l+1} \mathbf{p}_{0}^{l+1}+A^{l} \mathbf{p}_{0}^{l}\right)\right)=\boldsymbol{\omega}^{T} \mathbf{p}_{0}^{l}=P_{0}^{l}=P_{0}^{0},
$$

by (29) and conservation. The total discrete probability in (34) and the total probability in (13) share the same property: conservation over time. The total discrete probability of $\mathbf{p}_{k}^{l+1}$ is $\boldsymbol{\omega}^{T} \mathbf{p}_{k}^{l+1}$ and satisfies (33). 
The nonlinear system of equations to be solved for $\mathbf{p}_{0}^{l+1}, \mathbf{p}_{k}^{l+1}, k=1, \ldots, \nu$, and $\bar{\phi}_{k}^{l+1}, k=\nu+1, \ldots, n$, is with $C^{l+1}=I-0.5 \Delta t A^{l+1}$

$$
\left(\begin{array}{ccccccc}
C^{l+1} & 0 & \ldots & & & & \\
0.5 \Delta t B_{1}^{l+1} & C^{l+1} & \ldots & 0 & & & \\
\vdots & & \ddots & & & & \\
0.5 \Delta t B_{\nu}^{l+1} & 0 & \ldots & C^{l+1} & 0 & \ldots & \\
0.5 \Delta t \boldsymbol{\omega}^{T} B_{\nu+1}^{l+1} & 0 & \ldots & 0 & 1 & \ldots & 0 \\
\vdots & \vdots & & \vdots & & \ddots & \\
0.5 \Delta t \boldsymbol{\omega}^{T} B_{n}^{l+1} & 0 & \ldots & 0 & 0 & \ldots & 1
\end{array}\right)\left(\begin{array}{c}
\mathbf{p}_{0}^{l+1} \\
\mathbf{p}_{1}^{l+1} \\
\vdots \\
\mathbf{p}_{\nu}^{l+1} \\
\bar{\phi}_{\nu+1}^{l+1} \\
\vdots \\
\bar{\phi}_{n}^{l+1}
\end{array}\right)=\mathbf{b}^{l}
$$

where the right hand side $\mathbf{b}^{l}$ depends on the solution at $t^{l}$. The equations (35) are solved by a Newton-Krylov method [17] with GMRES [24] as the basic iterative method. With $L$ cells in each coordinate direction, the number of unknowns is $L^{m}(\nu+1)+(n-\nu)$. The original $N$-dimensional problem with the same discretization has $L^{m+n}$ unknowns. A reduction by about $L^{-n}$ has been accomplished.

\section{Properties of the solutions}

If there is a negative component of $\boldsymbol{\phi}$ in $w_{r}(\mathbf{x}, \boldsymbol{\phi}, t), w_{r}$ may become negative for some $r$ and there is a risk of a severe instability in the numerical solution. Since $\boldsymbol{\phi}$ is determined by $\bar{\phi}_{k}$ and $\mathbf{p}_{k}$ and $\mathbf{p}_{0}$ in (30), it is important to preserve the nonnegativity of the solutions $\mathbf{p}_{k}$ and $\bar{\phi}_{k}$ of (32) and (33) and the positivity of $\mathbf{p}_{0}$ in (29). The analytical solutions of (5), (11), and (7) have these properties under certain conditions. Sufficient conditions are derived for the numerical solutions to be non-negative and positive too.

Other algorithms to maintain the non-negativity of solutions of ODEs are discussed in [25]. One alternative there if the sign constraint on the solution is violated is to halve the time step and recompute the solution. The convectiondiffusion-reaction equation in one dimension is solved in [19] with finite difference and finite element methods. Bounds are derived on the time step and the Courant number for positive solutions. A scalar hyperbolic PDE in one dimension is solved in [3] with a finite element method. By choosing the coefficients in the space discretization depending on the solution, it remains positive. We will show here that our scheme in Section 3 also preserves non-negativity and positivity by taking sufficiently small time steps. The alternative in [25] to reset the negative variable to zero is not satisfactory for $\mathbf{p}_{0}$. By letting $p_{0 \mu}^{l}:=\max \left(\varepsilon, p_{0 \mu}^{l}\right), \phi_{k \mu}^{l}$ depends critically on the choice of $\varepsilon>0$.

Consider equations (11), (5), and (7) separate from each other without the coupling via $\phi$ and $p_{0}$. Let

$$
\hat{w}_{k}(\mathbf{x}, \boldsymbol{\phi}, t)=-\sum_{r} n_{r k} w_{r}(\mathbf{x}, \boldsymbol{\phi}, t)
$$


and assume that $\hat{w}_{k}$ and $\phi$ are in $C^{1}$ with respect to the independent variables. Then we have the following result for the analytical solution $\bar{\phi}_{k}, k=\nu+1, \ldots, n$, of $(7)$.

Theorem 1. Assume that for $k=\nu+1, \ldots, n$, and $t \geq 0$

$$
\hat{w}_{k}\left(\mathbf{x}, \phi_{1}, \ldots, \phi_{\nu}, \bar{\phi}_{\nu+1}, \ldots, \bar{\phi}_{k-1}, 0, \bar{\phi}_{k+1}, \ldots, \bar{\phi}_{n}, t\right) \geq 0,
$$

when $\bar{\phi}_{j} \geq 0, j \neq k, p_{0}(\mathbf{x}, t) \geq 0$, and that $\bar{\phi}_{k}(0) \geq 0$. Then

$$
\bar{\phi}_{k}(t) \geq 0
$$

when $t>0$.

Proof. Let $\tilde{w}_{k}(\boldsymbol{\phi}, t)=\int_{\mathbb{R}_{+}^{m}} \hat{w}_{k}(\mathbf{x}, \boldsymbol{\phi}, t) p_{0}(\mathbf{x}, t) d \mathbf{x}$. Then

$$
\tilde{w}_{k}\left(\phi_{1}, \ldots, \phi_{\nu}, \bar{\phi}_{\nu+1}, \ldots, \bar{\phi}_{k-1}, 0, \bar{\phi}_{k+1}, \ldots, \bar{\phi}_{n}, t\right) \geq 0 \text {. }
$$

If at some instance $t_{0}$ we have $\bar{\phi}_{k}\left(t_{0}\right)=0$, then in $(7) d \bar{\phi}_{k} / d t=\tilde{w}_{k} \geq 0$ and $\bar{\phi}_{k}$ can never cross the boundary $\bar{\phi}_{k}=0$ and become negative.

For the numerical solution of (7) we have a similar result.

Theorem 2. Assume that (37) is satisfied, $\mathbf{p}_{0}^{l} \geq 0$ for all $l$, and that $\bar{\phi}_{k}^{0}>0$ for $k=\nu+1, \ldots, n$. Let $\Delta t$ be sufficiently small. Then

$$
\bar{\phi}_{k}^{l}>0
$$

when $l>0$.

Proof. Assume that $\bar{\phi}^{l}>0$. Then compute $\bar{\phi}^{l+1}$ and we will show that $\bar{\phi}^{l+1}>0$.

Let $\tilde{w}_{k}^{l}=-\boldsymbol{\omega}^{T} B_{k}^{l} \mathbf{p}_{0}^{l}$ in (33). For $\bar{\phi}_{k}^{l+1}$ in (7) we have that

$$
\bar{\phi}_{k}^{l+1}=\bar{\phi}_{k}^{l}+0.5 \Delta t \tilde{w}_{k}^{l+1}\left(\bar{\phi}^{l+1}\right)+0.5 \Delta t \tilde{w}_{k}^{l}\left(\bar{\phi}^{l}\right) .
$$

There is a bound $\Delta t_{1}$ such that $\bar{\phi}_{k}^{l}+0.5 \Delta t \tilde{w}_{k}^{l}\left(\bar{\phi}^{l}\right)>0$ when $\Delta t \leq \Delta t_{1}$ since $\bar{\phi}_{k}^{l}>0, w_{k}^{l} \in C^{1}$, and $\tilde{w}_{k}^{l}\left(\phi_{1}^{l}, \ldots, \bar{\phi}_{k-1}^{l}, 0, \bar{\phi}_{k+1}^{l}, \ldots\right) \geq 0$.

Consider the index set $\mathcal{K}$ consisting of all $k$ such that $\left|\bar{\phi}_{k}^{l+1}\right| \leq \varepsilon$ for some small $\varepsilon>0$ for which there is a risk that $\bar{\phi}_{k}^{l+1}$ is non-positive. Partition $\bar{\phi}^{l+1}$ such that $\bar{\phi}_{I}$ consists of components with $k \in \mathcal{K}$ and $\bar{\phi}_{I I}$ holds the remaining components. For $\tilde{w}_{k}^{l+1}\left(\bar{\phi}^{l+1}\right), k \in \mathcal{K}$, we have the Taylor expansion about $\bar{\phi}_{I}^{l+1}=0$

$$
\tilde{\mathbf{w}}_{I}^{l+1}\left(\bar{\phi}^{l+1}\right)=\tilde{\mathbf{w}}_{0}^{l+1}\left(\overline{\boldsymbol{\phi}}_{I I}^{l+1}\right)+W^{l+1} \overline{\boldsymbol{\phi}}_{I}^{l+1},
$$


where $W_{i j}^{l+1}$ depends on $\partial \tilde{w}_{i} / \partial \phi_{j}$, see p. 190 in [5], and $\tilde{\mathbf{w}}_{0}^{l+1} \geq 0$. The solution $\bar{\phi}_{I}^{l+1}$ satisfies

$$
(D-0.5 \Delta t B) \bar{\phi}_{I}^{l+1}=\mathbf{c}
$$

where $D=\operatorname{diag}\left(1-0.5 \Delta t W_{j j}^{l+1}\right), B_{i i}=0, B_{i j}=W_{i j}^{l+1}$ when $i \neq j$, and $\mathbf{c}>0$. Thus,

$$
\bar{\phi}_{I}^{l+1}=\left(I-0.5 \Delta t D^{-1} B\right)^{-1} D^{-1} \mathbf{c} .
$$

If at least one $W_{j j}^{l+1}$ is positive, then a bound $\Delta t_{2}=2 / \max _{i} \max \left(W_{i i}^{l+1}, 0\right)$ on $\Delta t$ guarantees that $D$ is invertible and has positive diagonal elements. If all $W_{j j}^{l+1}$ are non-positive, then any positive $\Delta t$ is acceptable. Let $\Delta t_{3}<\Delta t_{2}$ be such that

$$
0.5 \Delta t_{3}\left\|D^{-1} B\right\|_{\infty}=0.5 \Delta t_{3} \max _{i}\left|1-0.5 \Delta t_{3} W_{i i}^{l+1}\right|^{-1} \sum_{j, j \neq i}\left|W_{i j}^{l+1}\right|<1 .
$$

The right hand side in (38) is

$$
\left(I-0.5 \Delta t D^{-1} B\right)^{-1} D^{-1} \mathbf{c}=D^{-1} \mathbf{c}+\left(\left(I-0.5 \Delta t D^{-1} B\right)^{-1}-I\right) D^{-1} \mathbf{c},
$$

with $D^{-1} \mathbf{c}>0$. For $\Delta t \leq \Delta t_{3}$ we have

$$
\begin{aligned}
& \left\|\left(I-0.5 \Delta t D^{-1} B\right)^{-1}-I\right\|_{\infty} \leq \\
& \sum_{j=1}^{\infty} 0.5^{j} \Delta t^{j}\left\|D^{-1} B\right\|_{\infty}^{j}=0.5 \Delta t\left\|D^{-1} B\right\|_{\infty} /\left(1-0.5 \Delta t\left\|D^{-1} B\right\|_{\infty}\right) .
\end{aligned}
$$

Choose $\Delta t_{4} \leq \Delta t_{3}$ such that

$$
0.5 \Delta t_{4}\left\|D^{-1} B\right\|_{\infty} /\left(1-0.5 \Delta t_{4}\left\|D^{-1} B\right\|_{\infty}\right)\left\|D^{-1} \mathbf{c}\right\|_{\infty}<\min _{j}\left(D^{-1} \mathbf{c}\right)_{j} .
$$

Then the expression in (39) is positive. Therefore, if $\Delta t \leq \min \left(\Delta t_{1}, \Delta t_{4}\right)$ then $\phi^{l+1}>0$ in (38) and the theorem is proved.

The equations (5) for $p_{k}$ and (10) for $p_{0}$ are investigated by means of the maximum principle for parabolic equations to show that a positive initial solution will remain positive in a finite interval $(0, T]$. Let $M(\mathbf{x}, t) \in \mathbb{R}^{m \times m}$ have the elements

$$
M_{i j}=\sum_{r=1}^{R} m_{r i} m_{r j} w_{r}(\mathbf{x}, \boldsymbol{\phi}, t)
$$

so that the second order term in (5) and (10) can be written

$$
0.5 \sum_{i, j=1}^{m} M_{i j} \frac{\partial^{2} p}{\partial x_{i} \partial x_{j}}
$$


with $p=p_{0}$ or $p=p_{k}$. Then we have

Theorem 3. Assume that $M$ is positive definite and that $w_{r}$ and $\boldsymbol{\phi}$ are in $C^{2}$ in $\bar{\Omega}_{h} \times[0, T]$ in their independent variables.

If $p_{0}(\mathbf{x}, t) \geq 0$ on the boundary $\partial \Omega_{h}$ and the initial conditions are $p_{0}(\mathbf{x}, 0)>0$ in $\Omega_{h}$, then $p_{0}(\mathbf{x}, t)>0$ in $\Omega_{h} \times(0, T]$.

If $p_{k}(\mathbf{x}, t) \geq 0$ on the boundary $\partial \Omega_{h}$, the initial conditions are $p_{k}(\mathbf{x}, 0)>0$ in $\Omega_{h}$ for $k=1, \ldots, \nu, p_{0}(\mathbf{x}, t) \geq 0$, and $\hat{w}_{k}(\mathbf{x}, \phi, t) \geq 0$, then $p_{k}(\mathbf{x}, t)>0$ in $\Omega_{h} \times(0, T]$.

Proof. The strong minimum principle on p. 122 in [22] is applicable with $\inf _{\Omega \times(0, T]} p=0$ since $M$ is positive definite. If $p_{j}=0, j=0,1, \ldots, \nu$, somewhere in $\Omega_{h} \times(0, T]$ then $p_{j}=0$ everywhere, which contradicts the initial condition. Hence, $p(\mathbf{x}, t)>0$ in $\Omega_{h}$ for $t \in(0, T]$.

Remark. A sufficient condition for $M$ to be positive definite is that all $w_{r}$ are positive in $\bar{\Omega}_{h}$ and that the stoichiometric matrix

$$
S=\left(\mathbf{m}_{1}, \mathbf{m}_{2}, \ldots, \mathbf{m}_{R}\right) \in \mathbb{R}^{m \times R}
$$

has rank $m$ (see [26]). The propensities will be positive at least in $\Omega_{h}$ if $\phi$ is positive there.

The steady state solution of (11) satisfies an elliptic equation with $M_{i j}$ multiplying the second derivatives and

$$
c=\sum_{r=1}^{R}\left(\mathbf{m}_{r} \cdot \nabla\right)\left(w_{r}+0.5\left(\mathbf{m}_{r} \cdot \nabla\right) w_{r}\right)
$$

multiplying $p_{0}$ in (10). If $c \leq 0$, then the maximum (or minimum) principle on $\mathrm{p}$. 35 in [9] or p. 109 in [22] is applicable also to the steady state problem to show that a solution will be positive in the interior of the domain.

The solutions of (29) and (32) have properties similar to those of the solutions of (10) and (5).

Theorem 4. Assume that $w_{r \mu}^{l}=w_{r}\left(\mathbf{x}_{\mu}, \phi_{\mu}^{l}, t^{l}\right) \geq 0$ for all $l>0$ and that $\Delta t$ is sufficiently small.

If $p_{0 \mu}^{0}>0$ for every $\mu$, then $p_{0 \mu}^{l}>0$.

If $p_{k \mu}^{0}>0$ for every $\mu$ and $k=1, \ldots, \nu, p_{0 \mu}^{l} \geq 0$, and $\hat{w}_{k}\left(\mathbf{x}_{\mu}, \phi_{\mu}^{l}, t^{l}\right) \geq 0$, then $p_{k \mu}^{l}>0$.

Proof. From (29) and (32) we conclude that both $\mathbf{p}_{0}^{l+1}$ and $\mathbf{p}_{k}^{l+1}$ satisfy

$$
\left(1-0.5 \Delta t A_{i i}\right) p_{i}^{l+1}=0.5 \Delta t \sum_{j \neq i} A_{i j} p_{j}^{l+1}+\mathbf{c}
$$


where $A=A^{l+1}, \mathbf{p}=\mathbf{p}_{0}$ or $\mathbf{p}=\mathbf{p}_{k}$, and

$$
\begin{aligned}
& \mathbf{c}=\left(I+0.5 \Delta t A^{l}\right) \mathbf{p}^{l}, \quad \text { if } \mathbf{p}=\mathbf{p}_{0}, \\
& \mathbf{c}=\left(I+0.5 \Delta t A^{l}\right) \mathbf{p}^{l}-0.5 \Delta t\left(B_{k}^{l+1} \mathbf{p}_{0}^{l+1}+B_{k}^{l} \mathbf{p}_{0}^{l}\right), \quad \text { if } \mathbf{p}=\mathbf{p}_{k} .
\end{aligned}
$$

We will show by induction that if $\mathbf{p}^{l}>0$ then $\mathbf{p}^{l+1}>0$.

The elements of $A$ are given by (24), (25), and Section 3.2. There is a $\Delta t_{1}$ such that if $\Delta t \leq \Delta t_{1}$, then $\left(I+0.5 \Delta t A^{l}\right)_{i j} \geq 0$. By the assumption on $\mathbf{p}_{0}^{l}$ and $\hat{w}_{k}$ we have $B_{k}^{l} \mathbf{p}_{0}^{l} \leq 0$ for every $l$ and therefore $\mathbf{c}>0$. If $\mathcal{J}$ is the set of cells neighboring cell $i$, then $\left|A_{i i}-A_{i j}\right| \leq \kappa h, j \in \mathcal{J}$, for some $\kappa$ since $w_{r} \in C^{1}$ and $A_{i j}=0$ when $j$ is outside $\mathcal{J}$. Then there is a $\Delta t_{2}$ such that $0.5 \Delta t \sum_{j} A_{i j} \leq 1-0.5 \Delta t A_{i i}$ for all $i$ if $\Delta t \leq \Delta t_{2}$.

Thus, in the interior and in the boundary cells $p_{i}^{l+1}$ satisfies

$$
p_{i}^{l+1}>0.5 \Delta t \sum_{j} A_{i j} p_{j}^{l+1} /\left(1-0.5 \Delta t A_{i i}\right)
$$

Assume that $p_{i}^{l+1}=\min _{\mu} p_{\mu}^{l+1} \leq 0$. Then the lowest value of the right hand side in (41) is obtained by taking $p_{j}^{l+1}=p_{i}^{l+1}$ resulting in

$$
p_{i}^{l+1}>\left(0.5 \Delta t \sum_{j} A_{i j} /\left(1-0.5 \Delta t A_{i i}\right)\right) p_{i}^{l+1}
$$

and we have a contradiction unless $p_{i}^{l+1}>0$. Hence, if $\Delta t \leq \min \left(\Delta t_{1}, \Delta t_{2}\right)$, then $\mathbf{p}_{0}^{l+1}>0$ and $\mathbf{p}_{k}^{l+1}>0$ and the theorem is proved.

The steady state solution $\mathbf{p}_{0}^{\infty}$ of (29) satisfies

$$
A \mathbf{p}_{0}^{\infty}=0
$$

where $A=\lim _{l \rightarrow \infty} A^{l}$. There is one eigenvalue of $A$ equal to zero with the right eigenvector $\mathbf{p}_{0}^{\infty}$ and the left eigenvector $\boldsymbol{\omega}$ (see Section 3.3 and [8]). The properties of $\mathbf{p}_{0}^{\infty}$ are derived in the next theorem using Perron's theory for positive matrices. The directed graph of a matrix $A$ is defined by the elements of $A$. An $L \times L$ matrix has $L$ vertices in the graph and an edge between the vertices $i$ and $j$ if $A_{i j} \neq 0$. An irreducible matrix $A$ has a directed graph where there is a chain of edges between every pair of vertices in the graph (see [13]).

Theorem 6. With the space discretization in Section 3.1 assume that

$$
\lim _{l \rightarrow \infty} w_{r}\left(\mathbf{x}_{\mu}, \phi_{\mu}^{l}, t^{l}\right)=w_{r \mu}^{\infty} \geq 0
$$

and that all other eigenvalues of $A$ except 0 satisfy $\Re \lambda(A)<0$. Then $\mathbf{p}_{0}^{\infty} \geq 0$. If in addition $A$ is irreducible, then there is only one $\lambda(A)=0$ and $\mathbf{p}_{0}^{\infty}$ is unique and positive. 
Proof. For a sufficiently small $\gamma>0, I+\gamma A$ has non-negative elements and the eigenvalue of largest modulus is 1 . The corresponding eigenvector $\mathbf{v}$ is the Perron vector and fulfills $\mathbf{v} \geq 0$ according to p. 158 in [13]. We have $A \mathbf{v}=0$ and $\mathbf{p}_{0}^{\infty}=\mathbf{v}$. If $A$ is irreducible, then $I+\gamma A$ has the same property. If $I+\gamma A$ is irreducible and has another eigenvalue with modulus 1 , then it is $\exp (i \psi)$ for some $\psi \neq 0$ with eigenvector $\mathbf{v}>0$ by the Perron-Frobenius theory (p. 159 in [13]). The corresponding $\lambda(A)=(\exp (i \psi)-1) / \gamma \neq 0$ and $\lambda(A)=0$ and $\mathbf{p}_{0}^{\infty}$ are unique.

Remark. As an example, a sufficient condition in one dimension for our matrix $A$ to be irreducible is $\hat{w}_{r j}^{\infty} \neq 0$ for all $j$. With the condition on $\lambda(A)$ in the theorem, all solutions of the ODE system $d \mathbf{p} / d t=A \mathbf{p}$ vanish when $t \rightarrow \infty$ except for $\mathbf{p}_{0}^{\infty}$. The condition on $c$ in (40) and on $\lambda(A)$ in the theorem are related in the following way: Decreasing $c$ would shift the spectrum of $A$ further into the left half plane.

If the conditions in Theorem 3 are fulfilled, then by (13) $p_{0}$ is constant in the $L_{1}$-norm for $t \geq 0$

$$
\left\|p_{0}(\cdot, t)\right\|_{1}=\int_{\Omega_{h}}\left|p_{0}(\mathbf{x}, t)\right| d \mathbf{x}=\int_{\Omega_{h}} p_{0}(\mathbf{x}, t) d \mathbf{x}=P_{0}(0) .
$$

The same property holds for $\mathbf{p}_{0}^{l}$ in the $\ell_{1}$-norm if the conditions in Theorem 4 are satisifed

$$
\left\|\mathbf{p}_{0}^{l}\right\|_{h, 1}=\sum_{\mu}\left|\omega_{\mu}\right|\left|p_{0 \mu}^{l}\right|=\sum_{\mu}\left|\omega_{\mu}\right| p_{0 \mu}^{l}=\omega^{T} \mathbf{p}_{0}^{0}=P_{0}^{0}
$$

according to (34).

\section{Numerical results for the circadian rhythm}

Living organisms have to adapt their behavior to different periodic changes in their environment e.g. the daily variation of light and the annual variation of temperature. Many of them have developed molecular clocks as their internal time-keeping mechanisms. An example of such an oscillatory process is the circadian rhythm with a period of about $24 \mathrm{~h}$ [12]. A model for this rhythm has been developed in [1] and is simplified in [28]. The reactions in this model are simulated here using the numerical method developed in the previous sections. With a particular choice of a parameter the macroscopic and deterministic model immediately arrives at a steady state solution but the mesoscopic and stochastic model continues to oscillate.

Consider the following 18 reactions for the nine molecular species 
A, $C, R, D_{a}, D_{a}^{\prime}, D_{r}, D_{r}^{\prime}, M_{a}, M_{a}^{\prime}$ modeling the circadian rhythm:

$$
\begin{aligned}
& \left.\left.\begin{array}{rcc}
D_{a}^{\prime} & \stackrel{\theta_{a} D_{a}^{\prime}}{\longrightarrow} & D_{a} \\
D_{a}+A & \stackrel{\gamma_{a} D_{a} A}{\longrightarrow} & D_{a}^{\prime} \\
D_{r}^{\prime} & \stackrel{\theta_{r} D_{r}^{\prime}}{\longrightarrow} & D_{r} \\
D_{r}+A & \stackrel{\gamma_{r} D_{r} A}{\longrightarrow} & D_{r}^{\prime}
\end{array}\right\} \begin{array}{ccc}
\emptyset & \stackrel{\alpha_{a}^{\prime} D_{a}^{\prime}}{\longrightarrow} & M_{a} \\
\emptyset & \stackrel{\alpha_{a} D_{a}}{\longrightarrow} & M_{a} \\
M_{a} & \stackrel{\delta_{m a} M_{a}}{\longrightarrow} & \emptyset
\end{array}\right\} \begin{array}{l}
\emptyset \\
\emptyset \\
\end{array} \\
& \left.\left.\begin{array}{rll}
\emptyset & \stackrel{\alpha_{r}^{\prime} D_{r}^{\prime}}{\longrightarrow} & M_{r} \\
\emptyset & \stackrel{\alpha_{r} D_{r}}{\longrightarrow} & M_{r} \\
M_{r} & \stackrel{\delta_{m r} M_{r}}{\longrightarrow} & \emptyset
\end{array}\right\} \begin{array}{ccc}
\emptyset & \stackrel{\beta_{r} M_{r}}{\longrightarrow} & R \\
R & \stackrel{\delta_{r} R}{\longrightarrow} & \emptyset \\
C & \stackrel{\delta_{a} C}{\longrightarrow} & R
\end{array}\right\}
\end{aligned}
$$

The reaction constants are found in Table 1 . The parameter $\delta_{r}$ will have two different values 0.2 and 0.08 in the numerical experiments.

\begin{tabular}{|c|c|c|c|c|c|c|c|c|c|}
\hline$\alpha_{a}$ & 50 & $\beta_{a}$ & 50 & $\gamma_{a}$ & 1 & $\delta_{m a}$ & 10 & $\theta_{a}$ & 50 \\
\hline$\alpha_{a}^{\prime}$ & 500 & $\beta_{r}$ & 5 & $\gamma_{r}$ & 1 & $\delta_{m r}$ & 0.5 & $\theta_{r}$ & 100 \\
\hline$\alpha_{r}$ & 0.01 & & & $\gamma_{c}$ & 2 & $\delta_{a}$ & 1 & & \\
\hline$\alpha_{r}^{\prime}$ & 50 & & & & & $\delta_{r}$ & - & & \\
\hline
\end{tabular}

Table 1: Parameters of the circadian clock (43).

Without any assumptions on the distributions of the molecular species, the FPE for (43) is a scalar PDE in nine dimensions for the PDF $p$ with $m=9, \nu=$ $0, n=0$. The computational complexity of the problem is reduced in two ways:

1. Complex $C$ and repressor $R$ are stochastic variables, the remaining species have normal distributions with a small $\sigma$, activator $A$ has a variable $\phi_{A}(\mathbf{x}, t)$ while the other expected values $\phi_{k}$ are only dependent on time $\Rightarrow 2$ PDEs in $2 \mathrm{D}, 6$ IDEs $(m=2, \nu=1, n=7)$.

2. All species have normal distributions with small $\sigma \Rightarrow$ reaction rate equations (8), 9 ODEs $(m=0, \nu=0, n=9)$.

In the first case, (11) determines $p_{0},(5)$ is solved for $p_{1}=p_{A}$ and $\phi_{A}(\mathbf{x}, t)$, and the expected values for the remaining six species satisfy $(7)$. In the second case, the equations are solved with the ODE integrator ode15s from MATLAB.

The initial values at $t=0$ are $D_{a}(0)=0.1, D_{r}(0)=0.1, D_{a}^{\prime}(0)=0.1, D_{r}^{\prime}(0)=$ $0.1, M_{a}(0)=0.1, M_{r}(0)=0.1$. After a short transient phase, the variables are adjusted so that the solution is in the periodic regime. The expected value $\phi_{A}$ is 0.1 initially and

$$
p_{0}(\mathbf{x}, 0)=\frac{1}{2 \pi \cdot 720} \exp \left(-\frac{\left(x_{1}-90\right)^{2}+\left(x_{2}-90\right)^{2}}{2 \cdot 720}\right) .
$$


The chemical system with $\delta_{R}=0.2$ is simulated in Figures 1 and 2 using the two modeling alternatives. The solution is computed with the reaction rate equations and displayed for the $D$ and $M$ variables in Figure 1. The solution with the stochastic model is similar. In Figure 2, the expected values of the complex and the repressor are compared to the solutions with the reaction rate equations. The periods with the two methods are almost the same with the ODE solution lagging slightly behind. Both solutions are oscillatory.

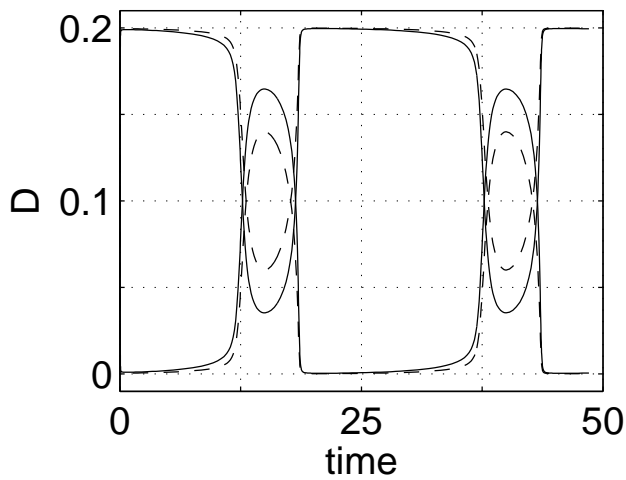

(a)

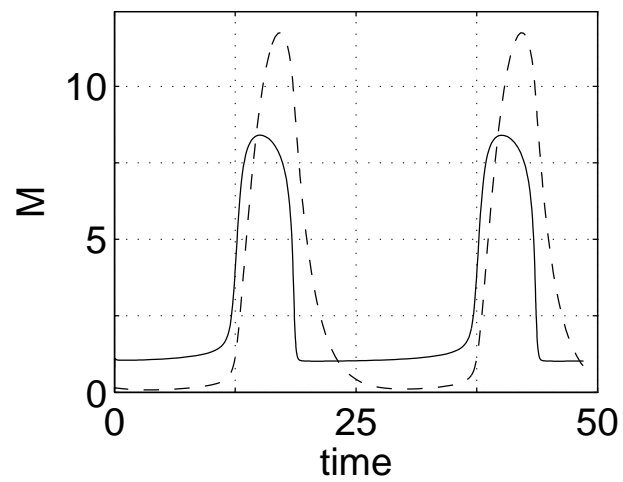

(b)

Figure 1: The solution is obtained by solving the ODEs for $\delta_{r}=0.2$. The species $D_{a}$ (solid upper solution at $\left.t \approx 1\right), D_{r}$ (dashed upper solution at $\left.t \approx 1\right), D_{a}^{\prime}$ (solid lower solution at $t \approx 1$ ), and $D_{r}^{\prime}$ (dashed lower solution at $t \approx 1$ ) are displayed in (a), and the species $M_{a}$ (solid) and $M_{r}$ (dashed) are displayed in (b).

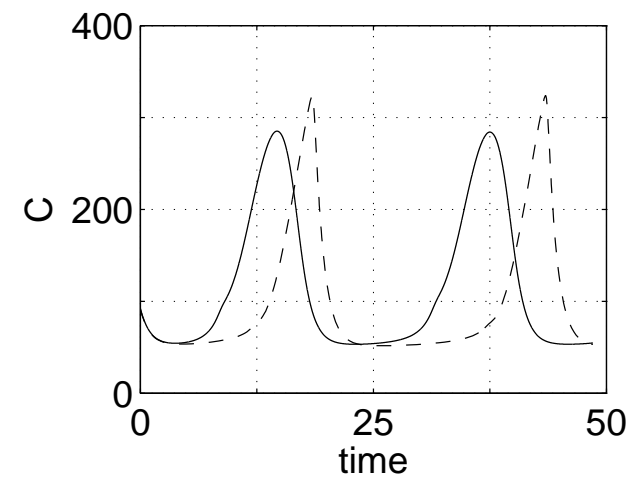

(a)

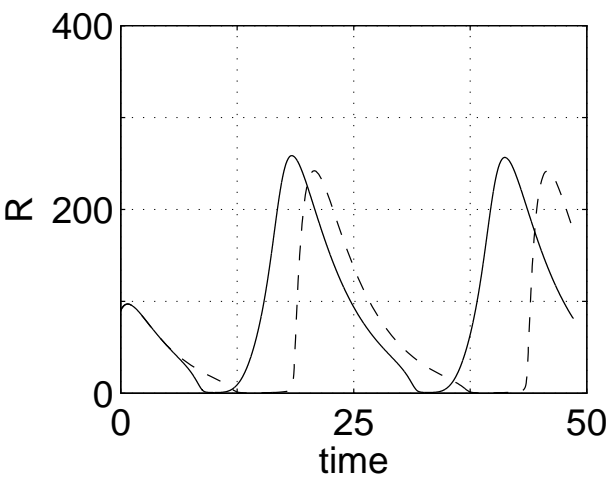

(b)

Figure 2: The expected values of $C$ (a) and $R$ (b) determined by $p_{0}$ (solid) and the concentrations of $C$ and $R$ obtained by the ODEs (dashed) with $\delta_{r}=0.2$.

The parameter $\delta_{r}$ is changed to 0.08 and the simulations are repeated with the same inital data in Figures 3 and 4 . There is no oscillation in the deterministic 
model. The solution quickly reaches a stable fixed point but the noise in the stochastic model is sufficiently strong to permit an oscillatory solution. This is in agreement with simulations in [28] using the SSA [10] and a method based on the evolution of the moments in [7]. Our simplified model with 2 PDEs in 2D and 6 IDEs captures the correct behavior of the original 9D problem.
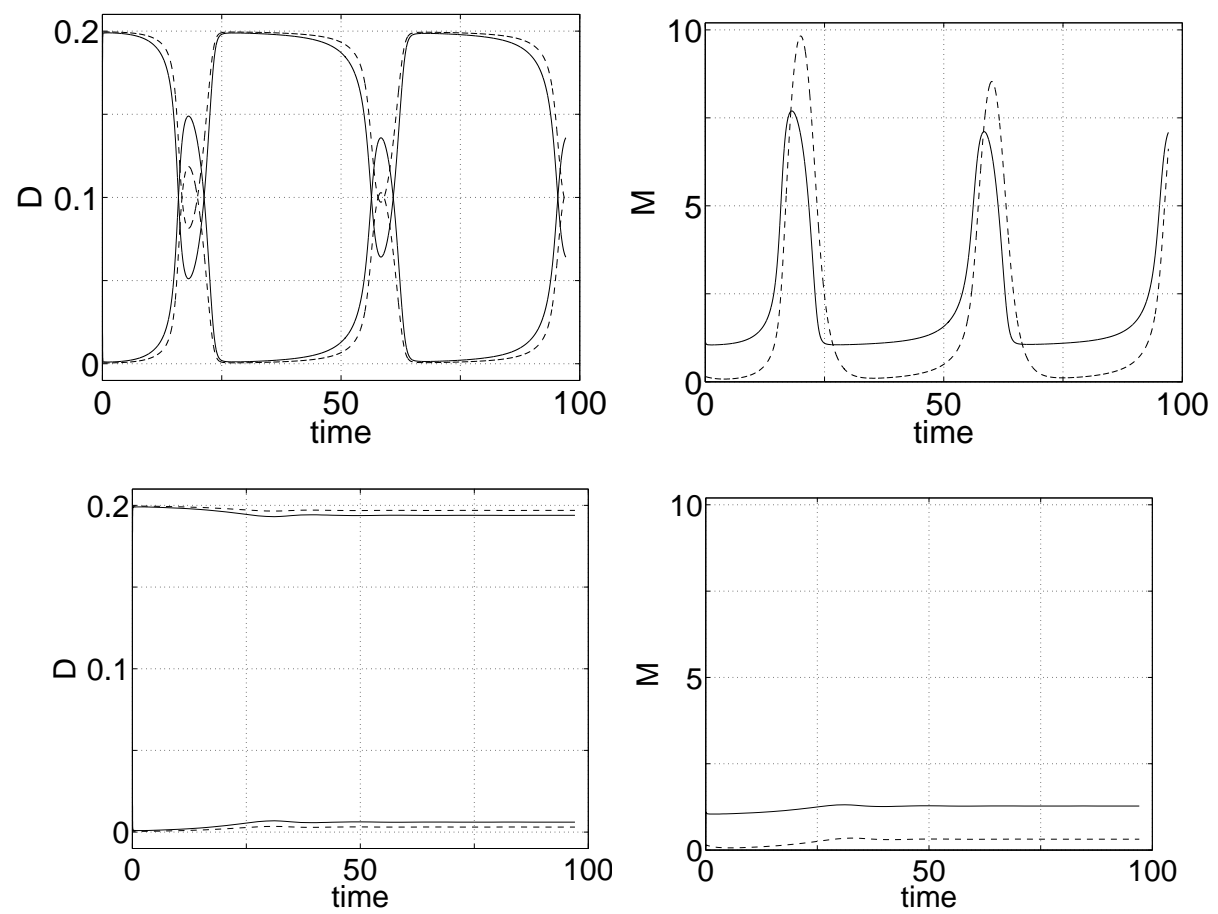

(a)

(b)

Figure 3: The solution is obtained by solving the IDEs (upper) and the ODEs (lower) for $\delta_{r}=0.08$. The species $D_{a}$ (solid upper solution at $\left.t \approx 1\right), D_{r}$ (dashed upper solution at $t \approx 1$ ), $D_{a}^{\prime}$ (solid lower solution at $t \approx 1$ ), and $D_{r}^{\prime}$ (dashed lower solution at $t \approx 1$ ) are displayed in column (a), and the species $M_{a}$ (solid) and $M_{r}$ (dashed) are displayed in column (b). 


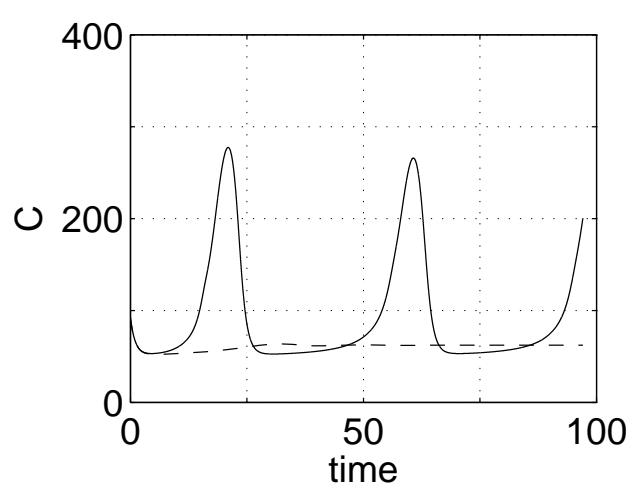

(a)

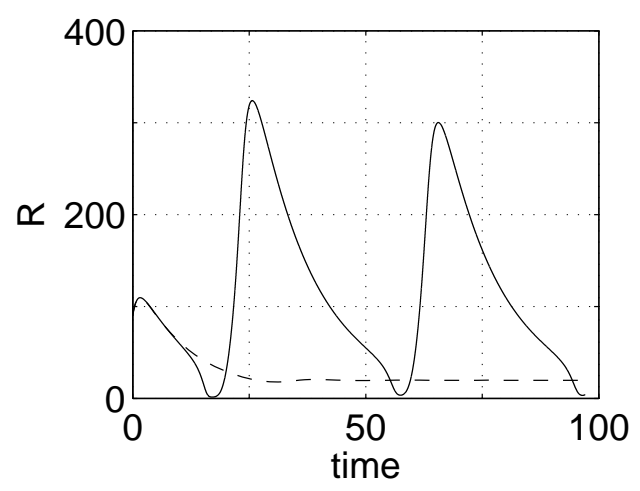

(b)

Figure 4: The expected values of $C$ (a) and $R$ (b) determined by $p_{0}$ (solid) and the concentrations of $C$ and $R$ obtained by the ODEs (dashed) with $\delta_{r}=0.08$.

The expected values of $p_{0}$ as a function of $C$ and $R$ and the $C$ and $R$ obtained with the reaction rate equations are also compared in Figure 5 for the two different $\delta_{r}$.

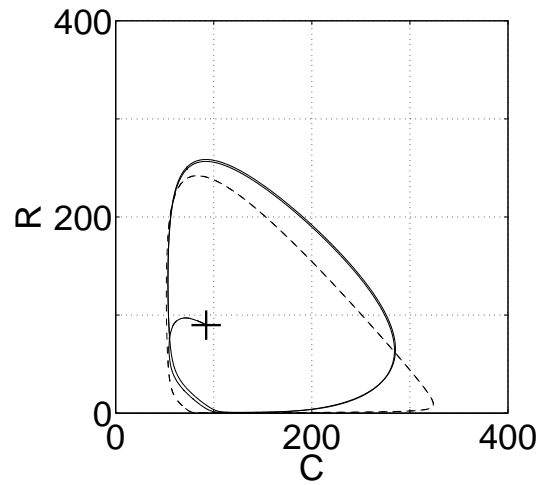

(a)

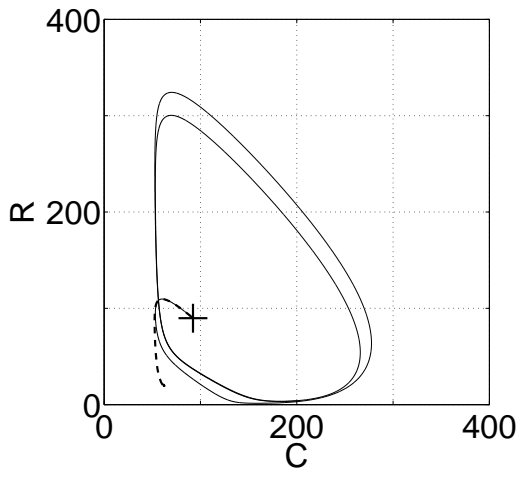

(b)

Figure 5: Two periods of the trajectory of the expected value of $p_{0}$ (solid) in the $C-R$ plane starting at + at $t=0$ and the trajectory computed with the ODEs (dashed). The parameter $\delta_{r}$ is 0.2 (a) and 0.08 (b).

The isolines of $p_{0}$ are shown for one period in Figure 6 . The solution rotates in the counterclockwise direction, spending most of the time along the line $C \approx 50$. 

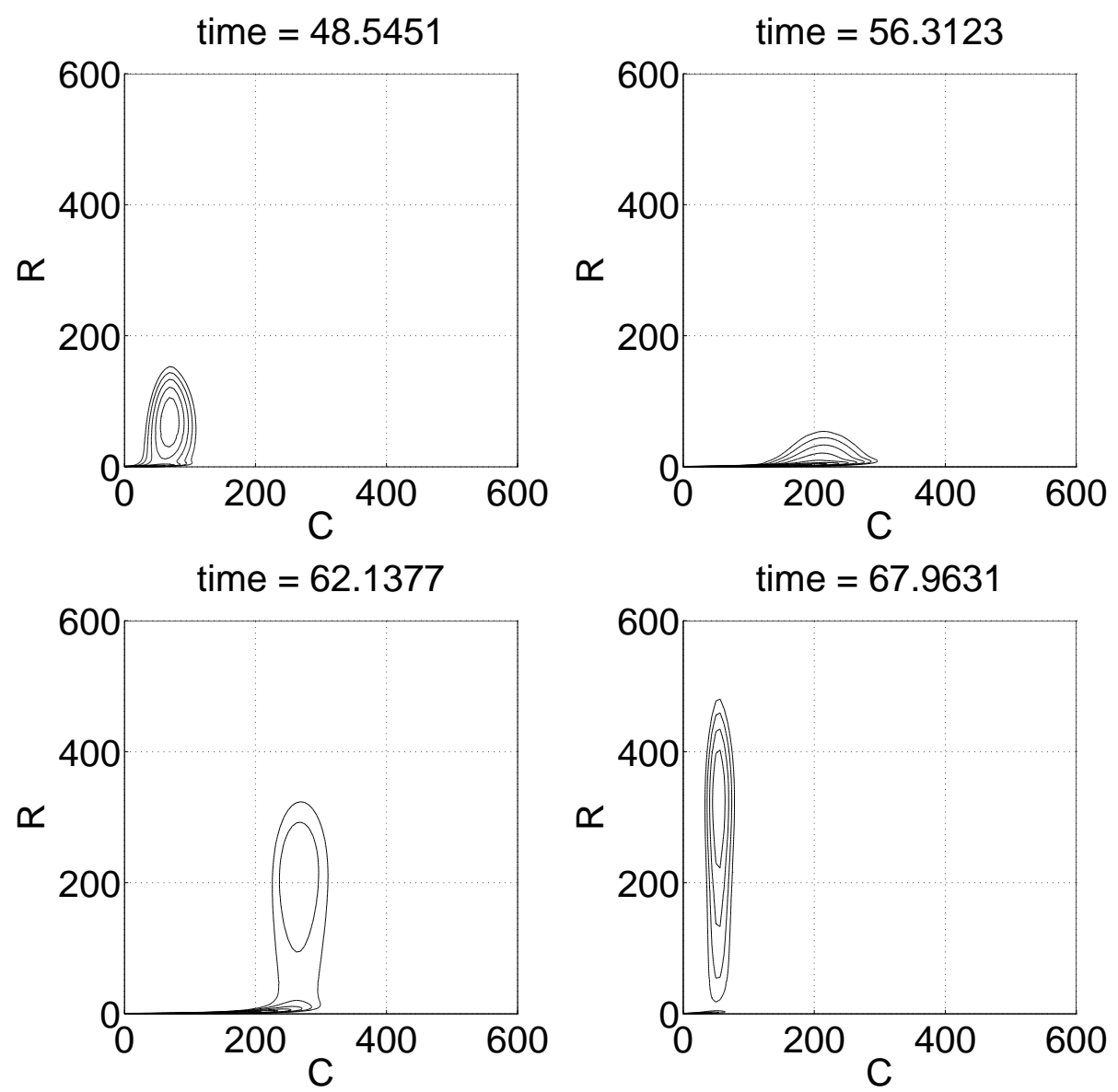

Figure 6: Isolines of $p_{0}$ with $\delta_{r}=0.08$ at $t=48.5,56.3,62.1$, and 68.0.

The activator solution is shown in Figure 7. The concentration is computed with the system of ODEs and the expected value is determined by the solution $p_{A}$ of (5). Assuming that $\phi_{A}$ is independent of $\mathbf{x}$ and introducing another IDE (7) for $\phi_{A}(t)$ instead of the PDE (5) is too inaccurate for sustained oscillations. The expected value of $A$ is far from being constant in $\mathbf{x}$-space and has support only close to the boundary where $R=0$. The concentration in the reaction rate equations is nonzero only for short time intervals, see Figure 7.

\section{Conclusions}

Chemical systems are modeled on a mesoscale with the Fokker-Planck equation. It is satisfied by the probability density of the number of molecules of the participating molecular species in the reactions. A reduced model is derived in [18] where the number of dimensions in the Fokker-Planck equation is lowered by assuming that some species have a normal distribution with a small variance. A numerical method to solve the reduced set of equations is presented. The method 
preserves the total probability and the non-negativity of the solution, properties shared by the analytical solution. The scheme is applied to a chemical model of a molecular clock commonly found in many organisms from bacteria to mammals. The oscillations are sensitive to a parameter in the model. If the parameter is sufficiently large then both the solutions from the macroscopic reaction rate equations and our reduced mixed macroscopic and mesoscopic model have an oscillatory behavior. With a lower value, only the solution with our reduced model continues to oscillate. This is in agreement with the conclusions in [28]. Furthermore, the computational complexity has been reduced from nine dimensions in the original problem to two dimensions.

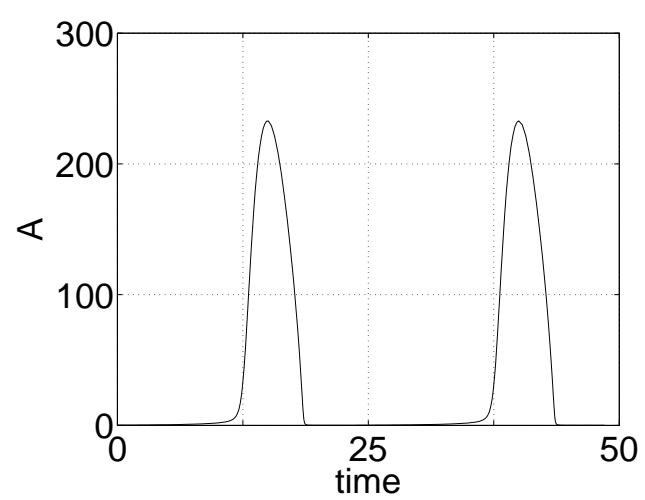

(a)

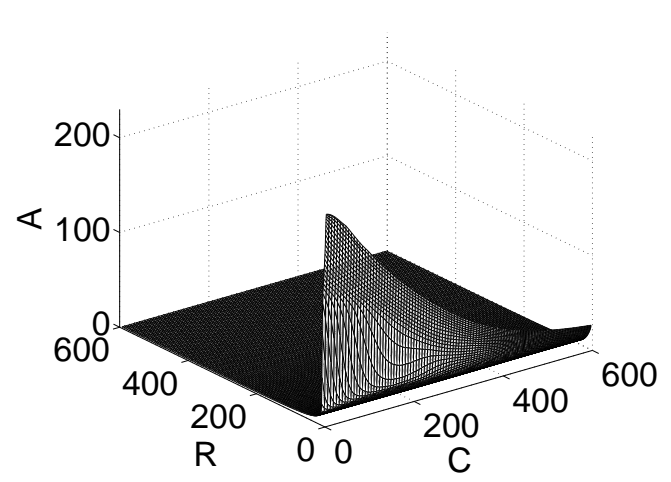

(b)

Figure 7: The concentration of $A$ determined by the ODEs and $\delta_{r}=0.2$ (a) and the expected value of $A, \phi_{A}(\mathbf{x}, T)$, at $T=97$ with $\delta_{r}=0.08$ (b).

\section{Acknowledgment}

This work has been supported by the Swedish Foundation for Strategic Research. Måns Ehrenberg and Johan Elf from the Department of Cell and Molecular Biology at Uppsala University have supplied us with the numerical example.

\section{References}

[1] N. Barkai, S. Leibler, Circadian clocks limited by noise, Nature, 403 (2000), p. 267-268.

[2] F. Baras, M. M. Mansour, Microscopic simulations of chemical instabilities, Adv. Chem. Phys., 100 (1997), p. 393-474.

[3] M. Berzins, Variable-order finite elements and positivity preservation for hyperbolic PDEs, Appl. Numer. Math., 48 (2004), p. 271-292. 
[4] Y. Cao, D. Gillespie, L. Petzold, Multiscale stochastic simulation algorithm with stochastic partial equilibrium assumption for chemically reacting systems, J. Comput. Phys., 206 (2005), p. 395-411.

[5] J. Dieudonné, Foundations of Modern Analysis, Academic Press, New York, 1969.

[6] J. Elf, J. Paulsson, O. G. Berg, M. Ehrenberg, Near-critical phenomena in intracellular metabolite pools, Biophys. J., 84 (2003), p. 154-170.

[7] S. EngBlom, Computing the moments of high dimensional solutions of the master equation, Technical report 2005-020, Dept of Information Technology, Uppsala University, Uppsala, Sweden, 2005, available at http://www.it.uu.se/research/reports/2005-020/, to appear in Appl. Math. Comput.

[8] L. Ferm, P. LÖtstedt, P. SJöBerG, Adaptive, conservative solution of the Fokker-Planck equation in molecular biology, Technical report 2004-054, Dept of Information Technology, Uppsala University, Uppsala, Sweden, 2004, available at http://www.it.uu.se/research/publications/reports/2004-054/.

[9] D. Gilbarg, N. S. Trudinger, Elliptic Partial Differential Equations of Second Order, Springer, Berlin, 1998.

[10] D. T. Gillespie, A general method for numerically simulating the stochastic time evolution of coupled chemical reactions, J. Comput. Phys., 22 (1976), p. $403-434$.

[11] D. T. GiLlespie, Exact stochastic simulation of coupled chemical reactions, J. Phys. Chem., 81 (1977), p. 2340-2361.

[12] A. Golbeter, Computational approaches to cellular rhythms, Nature, 420 (2002), p. 238-245.

[13] A. Greenbaum, Iterative Methods for Solving Linear Systems, SIAM, Philadelphia, 1997.

[14] E. Hairer, S. P. Nørsett, G. Wanner, Solving Ordinary Differential Equations, 2nd ed., Springer-Verlag, Berlin, 1993.

[15] E. Haseltine, J. Rawlings, Approximate simulation of coupled fast and slow reactions for stochastic chemical kinetics, J. Chem. Phys., 117 (2002), p. 6959-6969.

[16] N. G. van Kampen, Stochastic Processes in Physics and Chemistry, NorthHolland, Amsterdam, 1992. 
[17] D. A. Knolt, D. E. Keyes, Jacobian-free Newton-Krylov methods: a survey of approaches and applications, J. Comput. Phys., 193 (2004), p. $357-397$.

[18] P. Lötstedt, L. Ferm, Dimensional reduction of the Fokker-Planck equation for stochastic chemical reactions, Technical report 2005-023, Dept of Information Technology, Uppsala University, Uppsala, Sweden, 2005, available at http://www .it.uu. se/research/reports/2005-023/.

[19] R. J. MacKinnon, G. F. Carey, Positivity-preserving, flux-limited finitedifference and finite-element methods for reactive transport, Int. J. Numer. Meth. Fluids., 41 (2003), p. 151-183.

[20] H. H. McAdams, A. Arkin, It's a noisy business. Genetic regulation at the nanomolar scale, Trends Gen., 15 (1999), p. 65-69.

[21] C. V. RaO, A. P. Arkin, Stochastic chemical kinetics and the quasisteady-state assumption: Application to the Gillespie algorithm, J. Chem. Phys., 118 (2003), p. 4999-5010.

[22] M. Renardy, R. C. Rogers, An Introduction to Partial Differential Equations, Springer, New York, 1993.

[23] H. Risken, The Fokker-Planck Equation, 2nd ed., Springer, Berlin, 1996.

[24] Y. SAad, M. H. Schultz, GMRES: A generalized minimal residual algorithm for solving nonsymmetric linear systems, SIAM J. Sci. Stat. Comput., 7 (1986), p. 856-869.

[25] L. F. Shampine, S. Thompson, J. A. Kierzenka, G. D. Byrne, Nonnegative solutions of ODEs, Appl. Math. Comput., 170 (2005), p. 556-569.

[26] P. Sjöberg, P. Lötstedt, J. Elf, Fokker-Planck approximation of the master equation in molecular biology, Technical report 2005-044, Dept of Information Technology, Uppsala University, Uppsala, Sweden, 2005, available at http://www.it.uu.se/research/reports/2005-044/.

[27] M. Thattai, A. van Oudenaarden, Intrinsic noise in gene regulatory networks, Proc. Nat. Acad. Sci., 98 (2001), p. 8614-8619.

[28] J. M. G. Vilar, H. Y. Kueh, N. Barkai, S. Leibler, Mechanisms of noise-resistance in genetic oscillators, Proc. Nat. Acad. Sci., 99 (2002), p. 5988-5992. 